\title{
Two degrees of freedom rotating wall blocking sunlight directly tracking control model
}

\author{
Wang Shuying ${ }^{1, a^{*}}$, Wang $\mathrm{Yi}^{2, \mathrm{~b}}$ \\ ${ }^{1}$ Nanjing Institute of Industry Technology, China \\ ${ }^{2}$ School of computer science and technology Nanjing University of Science and Technology,China \\ awangsy@niit.edu.cn , b893762530@qq.com
}

Keywords: two degree of freedom, Wall block, Sunlight directly, Automatic tracking, Signal combination

Abstract. This paper expounds the necessity of sun tracking, the paper analyzes the straight line direction and plane around the essence of fixed-point movement, designs the light hitting the track vertical model, details model of the structure feature and functional performance, gives control equations and the corresponding relation table series, combined with the sun motion law ,the use of specific methods is given.

\section{Introduction}

Solar energy is a permanent topic of human studies, one of the core problem is to improve the solar energy conversion rate. In the illumination area must be under the condition of light conversion technology can't improve, improve the sun's rays point-blank time than is the key, According to the test, under the condition of same sunlight vertical irradiation is higher than natural exposure equipment capacity more than $35 \%{ }^{[1]}$, Due to the sun is clearance angle motion, request light equipment is always keep vertical state is more difficult with the sun. Using the tracking system of the market at present is mainly the preset program control and unidirectional uniform control machinery, the former needs more complex controller high cost of artificial control factors, the control accuracy is not high, to avoid bad weather function is poor, with a continuous run of high failure rate. Using plane and the normal relationship, this paper designs the automatic light point-blank tracking system, it is composed of stents, two-dimensional independent rotating mechanism, light plane, parallel to the wall block type signal receiver, and other components of the drive controller, With the method of combining theory with physical model, the plane around the fixed point motion, light surface two degree of freedom rotational and cuboids open container wall side light incident angle to unify, establish a list of control equations and the corresponding relation of each link, sunlight for specific position tracking, detailed settings and control methods are presented. This device has simple structure, low cost, convenient installation, high applicability, equipped with the clock function, can automatically return or avoid bad weather, prolong life, reduce energy consumption.

\section{The plane of fixed-point rotation.}

In space rectangular coordinate system ${ }^{0 x y z}$ (figure 1), straight line $L$ through the origin $\mathrm{O}$, direction angle for ${ }^{\alpha, \beta, \gamma}$ ( $L$ and three axis angle $\left.{ }^{O x, O y, O z}\right)^{[2]}, \mathrm{P}$ is $L$ on a bit, the direction of the vector $\overrightarrow{O P}$ cosine of $\cos \alpha, \cos \beta, \cos \gamma$, and there $\operatorname{are}^{\cos ^{2} \alpha+\cos ^{2} \beta+\cos ^{2} \gamma=1}$.

Plane $\Pi$ over the origin $O$ and $\overrightarrow{O P}$ as normal vector, the equations of $x \cos \alpha+y \cos \beta+z \cos \gamma=0$

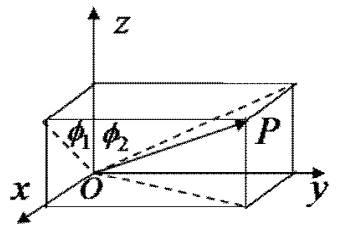

Figure 1. The vector and coordinate plane Angle.

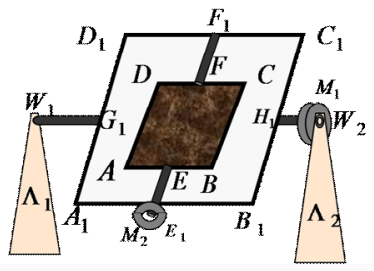

Figure 2. Independent rotating mechanism. 
Linear $L,{ }^{O y}$ have to plane ${ }^{y O P}$ with an angle coordinate plane ${ }^{y O z}$ is ${ }^{\phi_{1}}$, Linear $L, O x$ have to plane $x O P$ with an angle coordinate plane ${ }^{y O z}$ is ${ }^{\phi_{2}}$, and there are

$$
\tan \phi_{1}=\frac{\cos \alpha}{\cos \gamma}, \tan \phi_{2}=\frac{\cos \beta}{\cos \gamma}
$$

By type (1) can get the normal vector of plane $\Pi$ direction cosine of $\overrightarrow{O P}$ are as follows:

$$
\cos \alpha=\frac{ \pm \tan \phi_{1}}{\sqrt{\sec ^{2} \phi_{1} \sec ^{2} \phi_{1}+\tan ^{2} \phi_{1} \tan ^{2} \phi_{2}}}, \cos \beta=\frac{ \pm \tan \phi_{2}}{\sqrt{\sec ^{2} \phi_{1} \sec ^{2} \phi_{1}+\tan ^{2} \phi_{1} \tan ^{2} \phi_{2}}}, \cos \gamma=\frac{ \pm 1}{\sqrt{1+\tan ^{2} \phi_{1}+\tan ^{2} \phi_{2}}} \cdot
$$

Type (3) show that linear $L$ of direction angle is related to the plane angle. A coordinate axis ${ }^{O y}$ to $\left\{\cos \phi_{1}, 0, \sin \phi_{1}\right\}$ for the general equation of the normal vector of plane $\Pi_{1} x \cos \phi_{1}+z \sin \phi_{1}=0$, flat $\Pi_{1}$ line and perpendicular to the axis of the ${ }^{O y}$ on origin for ${ }^{L_{1}}$.

Set the $\vec{n}=\left(\alpha_{1}(t), \beta_{1}(t), \gamma_{1}(t)\right)$ is related to the parameter $t$ trend, With $\vec{n}$ in the normal vector at the origin of dynamic equations for planar $\Sigma$ :

$x \cos \alpha_{1}(t)+y \cos \beta_{1}(t)+z \cos \gamma_{1}(t)=0$.

If the plane of $\Sigma$ from equation for $z=0 \Pi_{0}$ (and coordinate plane ${ }^{x O y}$ overlap), ${ }^{O y}$ as the axis of rotation after ${ }^{\phi_{1}}$ and $\Pi_{1}$ overlap, plane angle ${ }^{\phi_{1}}$ called planar $\Pi_{1}$ in weft direction angle, ${ }^{o y}$ as zonal rotor; When the $\Sigma$ plane $\Pi_{1}$ from the beginning, $L_{1}$ as the axis of rotation after $\phi_{2}$ and $\Pi$ overlap, plane angle $\phi_{2}$ called planar $\Pi$ in warp direction angle, $L_{1}$ called meridian axis. Turn flat $\Sigma$ from $\Pi_{0}$ to $\Pi_{1}$ to Jackson's movement called planar $\Sigma$ orthogonal independent directional movement around the origin.

If a straight line of $L$ represents a light into the light of the origin, three coordinate surface are its incident plane surface (light) ${ }^{[3]}$, Light $L$ direction angle of ${ }^{\alpha, \beta, \gamma}$, respectively is the angle of incidence light on the surface of $y O z, z O x, x O y, L$ at the origin of the vertical plane is $\Pi$. If the light is in motion, the direction of the light $L$ angle is a function of the parameter $t(\alpha, \beta, \gamma)=(\alpha(t), \beta(t), \gamma(t))$, equation (3) is still the type, Called the plane $\Pi$ target plane, plane $\Sigma$ referred to as the track surface. When the luminous body movement, the light of $L(\alpha(t), \beta(t), \gamma(t))$ direction angle, the target plane $\Pi$ turn around the origin, tracking plane $\Sigma$ just keep consistent with the motion of plane of $\Pi$. Plane $\Sigma$ from $\Pi_{0}$ to $\Pi$ is the process of the fast track, in accordance with $\Pi$ process for synchronous tracking.

\section{Rotating mechanism}

Component combination of figure 2 are called orthogonal rotation mechanism, among them, the rectangular plane $A B C D$ installed inside a rectangular plane frame $A_{1} B_{1} C_{1} D_{1}, E_{1}, F_{1}, G_{1}, H_{1}$, in turn, is the midpoint of the border around the $A_{1} B_{1} C_{1} D_{1} A_{1} B_{1}, B_{1} C_{1}, C_{1} D_{1}, D_{1} A_{1}$, respectively fixed in $G_{1}, H_{1}$ a vertical axis of $A_{1} D_{1} G_{1} W_{1}, H_{1} W_{2}$, two axis $G_{1} W_{1}, H_{1} W_{2}$ in the same line, two axis respectively fixed to the bracket to the outer end of the ${ }^{\Lambda_{1}, \Lambda_{2}}$ at the top of the bearing, in motor ${ }^{M_{1}}$, mounted on the bracket ${ }^{\Lambda_{2}}$ motor are connected to the shaft $H_{1} W_{2} M_{1}$, motor ${ }^{M_{1}}$ rectangular frame ${ }^{A_{1} B_{1} C_{1} D_{1}} W_{1} W_{2}$ rotate around the axis rotation can be carried on. $E, F$, respectively is the midpoint of two parallel plane $A B C D$ edge $A B, C D$, respectively fixed at $E, F$ a vertical axis of $A B \quad E_{1} E, F_{1} F$, both the outer end of the shaft $E_{1} E, F_{1} F$ respectively fixed on the frame ${ }^{A_{1} B_{1} C_{1} D_{1}} E_{1}, F_{1}$ place bearing, two axis in the same line, in the framework of the $A B \quad E_{1} \quad M_{2}$ equipped with motor, the motor are connected to the shaft $E_{1} E M_{2}$, motor ${ }^{M_{2}}$ can drive axis rotation ${ }^{E_{1} E}$ rotation.

Stents $\Lambda_{1}, \Lambda_{2}$ fixed to the center of the rectangular planar frame ${ }_{1} B_{1} C_{1} D_{1}$ for origin $O$ establish the coordinate system ${ }^{O x y z}$ in figure 1, axial misalignments ${ }^{{ }^{+}{ }^{+}}$and ${ }_{1} W_{2}, O x^{+}, O y^{+}, O z^{+}$vertical shaft, plane $A B C D$ is the dynamic equation for (4) plane $\Sigma$. $A B C D$ and $A_{1} B_{1} C_{1} D_{1}$ when $t=0$ and coordinate plane $x O y$ overlap, axis of $E_{1} F_{1}$ on axis $O x$, plane $A B C D$ is $\Pi_{0}$. Start the motor $M_{1}(t>0)$, let the $A B C D$ with $A_{1} B_{1} C_{1} D_{1}$ closed after the rotation angle of $\phi_{1}\left(t=t_{1}\right)$, fixed ${ }^{A_{1} B_{1} C_{1} D_{1}}$, rectangle ${ }^{A_{1} B_{1} C_{1} D_{1}}$ 's plane is $\Pi_{1} .{ }_{1}$ axis $E_{1} F_{1}$ is the straight line. ${ }^{M_{2}}$, start the motor to the $A B C D$ around the axis of $E_{1} F_{1} \phi_{2}$ closed after $\left(t=t_{2}\right), A B C D$ is the plane equation (1) the plane of the $\Pi$.Obviously, tracking plane $A B C D$ for fast track, sets the 
angular velocity of the motor $M_{1}$ to ${ }^{\sigma_{11}}$, axis of $W_{1} W_{2}$ angular velocity $\omega_{11}=k_{1} \varpi_{11}$, motor $M_{2}$ angular velocity as the ${ }^{\omega_{21}}$, axis of $E_{1} F_{1}$ angular velocity as the $\omega_{21}=k_{2} \omega_{21}$, there are

$$
\phi_{2}=\omega_{22}\left(t_{2}-t_{1}\right)=k_{2} \varpi_{21}\left(t_{2}-t_{1}\right), \quad \phi_{1}=\omega_{12}\left(t_{1}-t_{0}\right)=k_{1} \varpi_{11}\left(t_{1}-t_{0}\right)
$$

Due to the axis of $W_{1} W_{2}, E_{1} F_{1}$ were independent of the vertical, the location of the plane $A B C D$ in $\Pi_{0}$ can start the motor $M_{1}$ and $M_{2}$, at the same time rotation respectively $\varpi_{11}\left(t_{1}-t_{0}\right), \varpi_{21}\left(t_{2}-t_{1}\right)$ can also arrived at the location of the $\Pi$, shortest tracking to ${ }^{\max }\left\{t_{1}-t_{0}, t_{2}-t_{1}\right\}$. The essence of this movement is the rotation of the two plane intersecting line, the coordinate plane $y O z \quad z O x \quad O y, O x$ axis separate rotating ${ }^{\phi_{1}}$, $\phi_{2}$, after the intersection of a turn before $O z$ as the rotation of $L$ line in a straight line.

\section{The signal receiver}

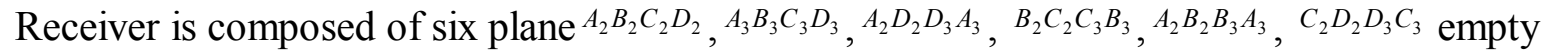
containers, round into rectangular shape $A_{2} B_{2} C_{2} D_{2}-A_{3} B_{3} C_{3} D_{3}$. the underside $A_{2} B_{2} C_{2} D_{2}$ is side length is $2 a$ square, four sides $A_{2} D_{2} D_{3} A_{3}, B_{2} C_{2} C_{3} B_{3}, A_{2} B_{2} B_{3} A_{3}, C_{2} D_{2} D_{3} C_{3}$ is wide for $2 a$ length of $h(h>2 a)$ rectangle, the five surface is made of opaque material sheet, the detailed specification sheet is coated with a layer of insulating material, the inside of each piece of thin plate with uniform photosensitive materials (or photosensitive components), photosensitive material can be light signal induction (current) ${ }^{[4]}$, the intersecting lines between adjacent two pieces of thin plate with insulation materials, can the independent signal light sheet called light sensor, with $G_{i}(i=1,2 \cdots, 5)$, in turn, said the plane $A_{2} B_{2} C_{2} D_{2}, A_{2} B_{2} B_{3} A_{3}, B_{2} C_{2} C_{3} B_{3}, C_{2} D_{2} D_{3} C_{3}, A_{2} D_{2} D_{3} A_{3}$ on the optical sensor, light sensor on each side to have separate wires to the output signal, on the underside ${ }_{3} B_{3} C_{3} D_{3}$ is made of transparent materials for $2 a$ square sheet.

Put the receiver down underside $A_{2} B_{2} C_{2} D_{2}$ fixed on the light side the $A B C D, A_{2} B_{2} C_{2} D_{2}$ center and the center of the $A B C D$ coincidence, two parallel side ${ }_{2} B_{2} B_{3} A_{3}, C_{2} D_{2} D_{3} C_{3}$ perpendicular to the axis ${ }_{1} F_{1}$, the

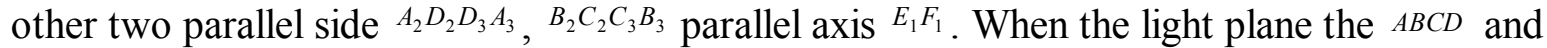
coordinate plane ${ }^{x O y}$ overlap, the side of the receiver and the coordinate surface ${ }^{y O z}, z O x$ parallel, light $L$ with each coordinate plane angle is equal to it and the receiver respectively inside plane angle.In receiver with $A B C D$ rotates, the light and the change of the wall angle is two rotation angle change. Set into the light and five light sensor on the surface of the ${ }^{G_{i}}$ in the angle of incidence of $0 \leq \theta_{i}<\pi / 2(i=1,2 \cdots, 5)$, otherwise is opposite value. Light sensor signal ${ }^{G_{i}}$ record for ${ }^{G_{i}}$ signal for $\overline{G_{i}}$, signal receiver combination ${ }^{G_{1}} \overline{G_{2}} \overline{G_{3} G_{4}} G_{5}$ said the light on the underside $A_{2} B_{2} C_{2} D_{2}$ sensillum of $G_{1}$ is light, four sides are not light.

That judgment with rectangle box structure light receiver called orthogonal parallel wall blocking the light receiver, the main features are: avoid light: when the light in the plane on the underside $A_{3} B_{3} C_{3} D_{3} \quad a_{11} x+a_{12} y+a_{13} z+d_{6}=0$ below, light can't into the receiver, the light sensor signal, signal into $\overline{G_{1} G_{2} G_{3} G_{4} G_{5}}\left(\theta_{i}<0(i=1,2 \cdots, 5)\right)$; Target tracking: in the light into and are parallel to the side, the vertical light irradiation on the underside, incidence angle $\theta_{1}=0, \theta_{i}=\pi / 2 \quad(i=2, \cdots, 5)$, only the light sensor ${ }^{G_{1}}$ signal, signal combination as $G_{1} \overline{G_{2}} \overline{G_{3} G_{4} G_{5}}$, tracking the target; Unipolarity: as the opaque to light barrier, on the inner wall of the light into the receiver, parallel to each other only a light sensor signal, such as side $A_{2} B_{2} B_{3} A_{3}$ illumination, $G_{2}$ all combinations of the signals $\overline{G_{1} G_{2}} \overline{G_{3}} \overline{G_{4}} \overline{G_{5}}, \overline{G_{1}} G_{2} G_{3} \overline{G_{4} G_{5}}, G_{1} G_{2} \overline{G_{3}} \overline{G_{4}} \overline{G_{5}}$, $\overline{G_{1}} G_{2} \overline{G_{3}} \bar{G}_{4} G_{5}, G_{1} G_{2} \overline{G_{3} G_{4}} G_{5}, G_{4}$ have no signal, show that with the side of the $A_{2} B_{2} B_{3} A_{3}$ parallel sides $C_{2} D_{2} D_{3} C_{3}$ no exposure. Receiver is the lining of the orthogonal parallel, each plane is represents a direction;Between adjacent light sensor signal combination: at least is perpendicular, parallel light that shoots through opening, five light sensor in at least one up to three by irradiation, was also illuminate the light sensor is two or more adjacent, signal combinations of less easy to program control. Signal used in the combination group a total of 18 , see table 1 . 
Table 1. Several photosensitive device with the combination of the signal.

\begin{tabular}{|c|c|}
\hline$n$ & $\left(G, G_{2}, G_{3}, G_{4}, G_{5}\right)$ \\
\hline 0 & $\overline{G_{1}} \overline{G_{2}} \bar{G}_{3} \overline{G_{4}} \bar{G}_{5}$ \\
\hline 1 & $G_{1} \bar{G}_{2} \bar{G}_{3} \bar{G}_{4} \bar{G}_{5}, \bar{G}_{1} G_{2} \bar{G}_{3} \bar{G}_{4} \bar{G}_{5}, \bar{G}_{1} \bar{G}_{2} G_{3} \bar{G}_{4} \bar{G}_{5}, \bar{G}_{1} \bar{G}_{2} \bar{G}_{3} G_{4} \bar{G}_{5}, \bar{G}_{1} \bar{G}_{2} \bar{G}_{3} \bar{G}_{4} G_{5}$ \\
\hline 3 & 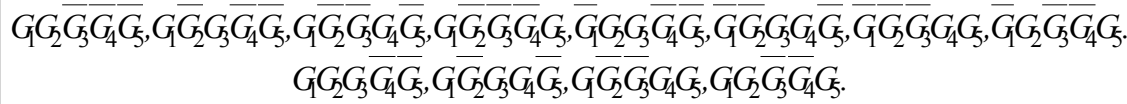 \\
\hline
\end{tabular}

Combination of signal value is by the position of the receiver and the angle of the light ${ }^{\theta_{i}}$, characterization of $0 \leq \theta_{i}<\pi / 2$ angle value of $\Phi\left(\theta_{i}\right)$, signal characterization of portfolio value for $f\left(G_{i}\right)$, table for the conversion between them

$$
f\left(G_{1}, G_{2}, G_{3}, G_{4}, G_{5}\right)=\Phi\left(\theta_{1}, \theta_{2}, \theta_{3}, \theta_{4}, \theta_{5}\right)
$$

Fantasy (8) the performance of the light angle and the relationship between signal combined with a given in table 2.

Table 2. The light angle and the combination of the signal.

\begin{tabular}{|c|c|}
\hline$\overline{\Phi\left(\theta_{1}, \theta_{2}, \theta_{3}, \theta_{4}, \theta_{5}\right)}$ & $f\left(G_{1}, G_{2}, G_{3}, G_{4}, G_{5}\right)$ \\
\hline$\theta_{i}<0$ & $\overline{G_{1} G_{2} G_{3} G_{4} G_{5}}$ \\
\hline$\theta_{1}=\pi / 2$ & $G_{1} \overline{G_{2}} \overline{G_{3}} \overline{G_{4} G_{5}}$ \\
\hline $\begin{array}{l}\theta_{3,5}=\pi / 2 \\
\theta_{2,4}=\pi / 2 .\end{array}$ & $\begin{array}{l}\bar{G}_{1} G_{2} \\
G_{1} \\
G_{2}\end{array}$ \\
\hline $0<\theta_{i}<\pi / 2$ & $\overline{G_{1}} G_{2} G_{3} \overline{G_{4}} \overline{G_{5}}, \overline{G_{1}} G_{2} G_{3} G_{4} \overline{G_{5}}, \overline{G_{1}} \overline{G_{2}} G_{3} G_{4} G_{5}, \overline{G_{1}} G_{2} \overline{G_{3}} G_{4} G_{5}, G_{1} G_{2} G_{3} \overline{G_{4}} \overline{G_{5}}, G_{1} \overline{G_{2}} G_{3} G_{4} \overline{G_{5}}, G_{1} \overline{G_{2} G_{3}} G_{4} G_{5}, G_{1} G_{2} \overline{G_{3}} \overline{G_{4}} G_{5}$ \\
\hline
\end{tabular}

\section{The controller}

Controller is made of electronic components and circuit boards and other microprocessors, or single chip microcomputer with auxiliary device is added, the input and the output of the receiver on the photosensitive device, output and the motor on the rotating mechanism ${ }^{M_{1}, M_{2}}$, motor ${ }_{1}, M_{2}$ can set various speed. Controller can convert electrical signals from the receiver to control signal output, outward $M_{1}, M_{2}$ control motor speed, the speed of a motor vector $V\left(v_{1}, v_{2}\right)$, it combined with signal representation for the transformation of the relationship between value $f\left(G_{i}\right)$ table

$$
V\left(v_{1}, v_{2}\right)=F\left(f\left(G_{1}, G_{2}, \cdots, G_{5}\right)\right)
$$

If the speed of the motor $M_{1}, M_{2}$ each set conventional speed $V_{0}\left(v_{10}, v_{20}\right)$, is fast $V_{1}\left(v_{11}, v_{21}\right)$ and $V_{2}\left(v_{12}, v_{22}\right)$ three levels in the opposite direction, the speed of the motor ${ }^{M_{1}}$ is $v_{10}, v_{11}, v_{12}$ in turn, the speed of the motor $M_{2}$ is $v_{20}, v_{21}, v_{22}$ in turn when the light on the receiver $G_{2} G_{3}$ motor for forward quickly, light on the receiver $G_{4} G_{5}$ motor as, in the opposite direction when the light is parallel to the side of the receiver for conventional speed when the motor, type (7) signal combination relationship with the motor speed are given in table 3.

\begin{tabular}{|c|c|c|}
\hline$f\left(G_{1}, G_{2}, G_{3}, G_{4}, G_{5}\right)$ & $v_{1}$ & $v_{2}$ \\
\hline$\overline{G_{1}} \overline{G_{2}} \overline{G_{3}} \overline{G_{4}} \overline{G_{5}}$ & 0 & 0 \\
\hline$G_{1} \overline{G_{2}} \overline{G_{3}} \overline{G_{4}} \overline{G_{5}}$ & $v_{10}$ & $v_{20}$ \\
\hline$\overline{G_{1}} G_{2} \overline{G_{3} G_{4}} \overline{G_{5}}, G_{1} G_{2} \overline{G_{3}} \overline{G_{4}} \overline{G_{5}}$ & $v_{11}$ & $v_{20}$ \\
\hline$\overline{G_{1}} \overline{G_{2}} G_{3} \overline{G_{4}} \overline{G_{5}}, G_{1} \overline{G_{2}} G_{3} \overline{G_{4}} \overline{G_{5}}$ & $v_{10}$ & $v_{21}$ \\
\hline$\overline{G_{1}} \overline{G_{2}} \overline{G_{3}} G_{4} \overline{G_{5}}, G_{1} \overline{G_{2}} \overline{G_{3}} G_{4} \overline{G_{5}}$ & $v_{12}$ & $v_{20}$ \\
\hline$\overline{G_{1}} \overline{G_{2}} \overline{G_{3}} \overline{G_{4}} G_{5}, G_{1} \overline{G_{2}} \overline{G_{3}} \overline{G_{4}} G_{5}$ & $v_{10}$ & $v_{22}$ \\
\hline$\overline{G_{1}} G_{2} G_{3} \overline{G_{4}} \overline{G_{5}}, G_{1} G_{2} G_{3} \overline{G_{4}} \overline{G_{5}}$ & $v_{11}$ & $v_{21}$ \\
\hline$\overline{G_{1}} \overline{G_{2}} G_{3} G_{4} \overline{G_{5}}, G_{1} \overline{G_{2}} G_{3} G_{4} \overline{G_{5}}$ & $v_{12}$ & $v_{21}$ \\
\hline$\overline{G_{1}} \overline{G_{2}} \overline{G_{3}} G_{4} G_{5}, G_{1} \overline{G_{2}} \overline{G_{3}} G_{4} G_{5}$ & $v_{11}$ & $v_{22}$ \\
\hline$G_{1} G_{2} G_{3} G_{4} G_{5}, G_{1} G_{2} G_{3} G_{4} G_{5}$ & $v_{12}$ & $v_{22}$ \\
\hline
\end{tabular}

Table 3. The combination and motor speed signal corresponding to the table

\section{The sun tracking control}

The independent rotating mechanism is equipped with signal receiver and controller called light tracking system, working process is: by the light incident angle $\theta_{i} f\left(G_{i}\right)$ different values form the signal combinations, after dealing with the controller as instruction signal, drive motor received instructions 
according to the set speed, motor rotation into independent axis of rotation, the rotation of the shaft to adjust light and the direction of the receiver, the light surface movement is the light direction angle with the target of control vector direction angle ${ }^{\alpha_{1}(t), \beta_{1}(t), \gamma_{1}(t) \alpha(t), \beta(t), \gamma(t)}$ differences, the movement of the receiver is to adjust the light incident angle change of ${ }^{\theta_{i}}$, such cycles to light the light with the target surface normal vector direction are consistent, comprehensive type (4) - (7) into circulation patterns for

$$
\theta_{i} \stackrel{\Phi\left(\theta_{i}\right)}{\longrightarrow} G_{i}\left(\theta_{i}\right) \stackrel{f\left(G_{i}\right)}{\longrightarrow} V\left(v_{1}, v_{2}\right) \stackrel{K\left(v_{j}\right)}{\longrightarrow} \omega\left(\omega_{1 k}, \omega_{2 k}\right) \stackrel{\left(\phi_{1}, \phi_{2}\right)}{\longrightarrow} \vec{n}\left(\alpha_{1}, \beta_{1}, \gamma_{1}\right) \rightarrow \theta_{i}(\vec{n})
$$

Comprehensive table 1-3 and (8) formed by various relationships listed in table 4.

Table 4. Combined with motor speed signal corresponding to the table

\begin{tabular}{|c|c|c|c|c|c|c|c|c|}
\hline$\left(\theta_{1}, \theta_{2}, \theta_{3}, \theta_{4}, \theta_{5}\right)$ & $f\left(G_{1}, G_{2}, G_{3}, G_{4}, G_{5}\right)$ & $v_{1}$ & $v_{2}$ & $\phi_{1}$ & $\phi_{2}$ & $\alpha_{1}$ & $\beta_{1}$ & $\gamma_{1}$ \\
\hline$<0$ & $\overline{G_{1}} \overline{G_{2}} \overline{G_{3}} \overline{G_{4}} \overline{G_{5}}$ & 0 & 0 & 0 & 0 & $\pi / 2$ & $\pi / 2$ & 0 \\
\hline$\theta_{1}=0$ & $G_{1} \overline{G_{2}} \overline{G_{3}} \overline{G_{4}} \overline{G_{5}}$ & $v_{10}$ & $v_{20}$ & $\omega_{10} t$ & $\omega_{20} t$ & $\alpha$ & $\beta$ & $\gamma$ \\
\hline$\theta_{3,5}=\pi / 2, \theta_{2} \neq \pi / 2$ & $G_{1} G_{2} G_{3} G_{4} G_{5}, G_{1} G_{2} G_{3} G_{4} G_{5}$ & $v_{11}$ & $v_{20}$ & $>0$ & 0 & $<\alpha$ & $\beta$ & $>\gamma$ \\
\hline$\theta_{2,4}=\pi / 2, \theta_{3} \neq \pi / 2$ & $\overline{G_{1}} \overline{G_{2}} G_{3} \overline{G_{4}} \overline{G_{5}}, G_{1} \overline{G_{2}} G_{3} \overline{G_{4}} \overline{G_{5}}$ & $v_{10}$ & $v_{21}$ & 0 & $<0$ & $\alpha$ & $<\beta$ & $>\gamma$ \\
\hline$\theta_{3,5}=\pi / 2, \theta_{4} \neq \pi / 2$ & $\overline{G_{1}} \overline{G_{2}} \overline{G_{3}} G_{4} \overline{G_{5}}, G_{1} \overline{G_{2}} \overline{G_{3}} G_{4} \overline{G_{5}}$ & $v_{12}$ & $v_{20}$ & $<0$ & 0 & $>\alpha$ & $\beta$ & $<\gamma$ \\
\hline$\theta_{3,5}=\pi / 2, \theta_{5} \neq \pi / 2$ & $\overline{G_{1}} G_{2} G_{3} \overline{G_{4}} G_{5}, G_{1} \overline{G_{2}} \overline{G_{3} G_{4}} G_{5}$ & $v_{10}$ & $v_{22}$ & 0 & $>0$ & $\alpha$ & $>\beta$ & $<\gamma$ \\
\hline $0<\theta_{1,2,3}<\pi / 2$ & $\overline{G_{1}} G_{2} G_{3} \overline{G_{4}} G_{5}, G_{1} G_{2} G_{3} \overline{G_{4}} \overline{G_{5}}$ & $v_{11}$ & $v_{21}$ & $>0$ & $<0$ & $<\alpha$ & $<\beta$ & $>\gamma$ \\
\hline $0<\theta_{1,3,4}<\pi / 2$ & $\overline{G_{1}} \overline{G_{2}} G_{3} G_{4} \overline{G_{5}}, G_{1} \overline{G_{2}} G_{3} G_{4} \overline{G_{5}}$ & $v_{12}$ & $v_{21}$ & $<0$ & $<0$ & $>\alpha$ & $<\beta$ & $<\gamma$ \\
\hline $0<\theta_{1,4,5}<\pi / 2$ & $G_{1} G_{2} G_{3} G_{4} G_{5}, G_{1} G_{2} G_{3} G_{4} G_{5}$ & $v_{11}$ & $v_{22}$ & $<0$ & $>0$ & $>\alpha$ & $>\beta$ & $<\gamma$ \\
\hline $0<\theta_{1,2,5}<\pi / 2$ & $\overline{G_{1}} G_{2} \overline{G_{3}} \overline{G_{4}} G_{5}, G_{1} G_{2} \overline{G_{3}} \overline{G_{4}} G_{5}$ & $v_{12}$ & $v_{22}$ & $>0$ & $>0$ & $<\alpha$ & $>\beta$ & $>\gamma$ \\
\hline
\end{tabular}

Table 4, the ${ }^{\omega_{10} t, \omega_{20} t}$ respectively lights around the point on the sphere movement of the weft to angle and meridional angle. $\phi_{1}>0, \phi_{2}<0$ for counterclockwise rotation.

Fantasy (8) and table 4 used in direct sunlight tracking control, it is convenient to be used in parameter identification and tracking. On the earth's surface where the sun tracking system, fixed light of $W_{1} W_{2}$ to east direction and horizontal zonal axis, receiver up. In the coordinate system ${ }^{O x y z}$ tracking system, the sun as a particle, the sun is linear $L$, the sun rises fall and seasonal variation of the earth, is the earth's rotation and orbit around the sun, show the straight line of $L$ in sphere by the motion of particles on and weft motion synthesis ${ }^{[5]}$, the dynamic linear $L$ around the origin angle to the warp and weft angle change. The sun were to rise in the fall velocity equal to the earth rotation angular velocity $\omega_{10}=2 \pi / T_{\beta}$, season of the sun movement speed is equal to the earth's orbital angular velocity $\left|\omega_{20}\right|=4 \gamma_{0} / T_{\alpha}$ ( $T_{\alpha},{ }_{\beta}$ earth's orbital period and rotation respectively, $\gamma_{0}$ for earth's equator and the ecliptic plane angle ${ }^{[5]}$ ).

System of synchronous tracking speed: conventional motor velocity vector: $V_{0}\left(v_{10}, v_{20}\right)=K\left(\omega_{10}, \omega_{20}\right)$. $\left(\omega_{10}=2 \pi / T_{\beta} \quad W_{1} W_{2}\right.$ for the rotation angular velocity, $\left|\omega_{20}\right|=4 \gamma_{0} / T_{\alpha} \quad E_{1} F_{1}$ for the rotation angular velocity).

System of fast tracking speed: Is the $V_{1}\left(v_{11}, v_{21}\right), V_{2}\left(v_{12}, v_{22}\right)$, the fast tracking speed of the motor, can consider system factors such as friction, load, motor power, recycling type (4) (5) to set.

System initialization setting: due to the discontinuous appear and the change in the weather, toward the ground of sunlight is intermittent, to save energy consumption to increase system lifetime, only set up in local work, when the sun did not stop work light, light angle of two adjacent to the largest $180^{\circ}$ (early and late), no interval of time when the weather is a dark night, when the weather interval of time, to facilitate the next job or the system failure can still maintain good radiation effect, can increase the clocks in the control system, in a row without light irradiation time through the system to return to the previous working day after noon.

\section{Conclusion}

Based on the characteristics of the plane and the normal, this paper analyzes the light around the point movement principle, designs the light plane rotation mechanism, the two degree of freedom parallel wall blocking the signal receiver and driving controller, sets up the light incident angle combination, signal conversion, drive mode, orthogonal axis angle and lighting series of plane rotation control equation, and by the list that give corresponding relations, combined with the feature of the sun 
to give further tracking control method, it is recommended to use the clock function sets the system initialization conditions.

\section{References}

[1]M. WeiXin Jiao, Hong Zou: Planetary science(Peking University press, China 2009)

[2]M.WenMao Yang, QuanYing Li:Space analytic geometry(Wuhan university press, China 2002).

[3]J. Shuying Wang: switzerland, Advanced Material Research .Vol.748 (2013) ,PP:811-814.

[4]M.Shensheng Li: An introduction to solar energy heat utilization (Higher education press, China1989).

[5]M. Su Yi: Introduction to astronomy new (science press ,China 2009). 